\title{
Optimization of molecularly targeted MRI in the brain: empirical comparison of sequences and particles
}

This article was published in the following Dove Press journal:

International Journal of Nanomedicine

\author{
Niloufar Zarghami' \\ Alexandre A Khrapitchev' \\ Francisco Perez-Balderas' \\ Manuel Sarmiento Soto' \\ James R Larkin' \\ Luca Bau² \\ Nicola R Sibson' \\ 'Cancer Research UK and Medical \\ Research Council Oxford Institute \\ for Radiation Oncology, Department \\ of Oncology, University of Oxford, \\ Oxford, UK; ${ }^{2}$ Institute of Biomedical \\ Engineering, Department of \\ Engineering Sciences, University \\ of Oxford, Oxford, UK
}

Background: Molecular MRI is an evolving field of research with strong translational potential. Selection of the appropriate MRI sequence, field strength and contrast agent depend largely on the application. The primary aims of the current study were to: 1) assess the sensitivity of different MRI sequences for detection of iron oxide particles in mouse brain; 2) determine the effect of magnetic field strength on detection of iron oxide particles in vivo; and 3) compare the sensitivity of targeted microparticles of iron oxide (MPIO) or ultra-small superparamagnetic iron oxide (USPIO) for detection of vascular cell adhesion molecule-1 (VCAM-1) in vivo.

Methods: Mice were injected intrastriatally with interleukin $1 \beta$ to induce VCAM-1 expression on the cerebral vasculature. Subsequently, animals were injected intravenously with either VCAM-MPIO or VCAM-USPIO and imaged 1 or 13 hours post-injection, respectively. MRI was performed at 4.7, 7.0, or $9.4 \mathrm{~T}$, using three different $T_{2}{ }^{*}$-weighted sequences: single gradient echo 3D (GE3D), multi-gradient echo 3D (MGE3D) and balanced steady-state free precession 3D (bSSFP3D).

Results: MGE3D yielded the highest signal-to-noise ratio (SNR) and contrast-to-noise ratio (CNR) for the detection of iron oxide particles. All sequences showed a significant increase in SNR and CNR from 4.7 to 7.0 T, but no further improvement at 9.4 T. However, whilst targeted MPIO enabled sensitive detection of VCAM-1 expression on the cerebral vasculature, the long half-life (16.5 h vs $1.2 \mathrm{~min})$ and lower relaxivity per particle $\left(1.29 \times 10^{-14} \mathrm{vs} 1.18 \times 10^{-9} \mathrm{~Hz}\right.$ L/particle) of USPIO vs. MPIO rendered them impractical for molecular MRI.

Conclusion: These findings demonstrate clear advantages of MPIO compared to USPIO for molecularly-targeted MRI, and indicate that the MGE3D sequence is optimal for MPIO detection. Moreover, higher field strengths (7.0/9.4 T) showed enhanced sensitivity over lower field strengths $(4.7 \mathrm{~T})$. With the development of biodegradable MPIO, these agents hold promise for clinical translation.

Keywords: molecular imaging, preclinical MRI, VCAM-MPIO, VCAM-USPIO, multi gradient echo, bSSFP

\section{Introduction}

Preclinical molecular and cellular MRI is an evolving field of research with strong translational potential. Iron oxide particles are currently the preferred contrast platform for this application and have been thoroughly reviewed in the literature. ${ }^{1-4}$ The magnetic core of these particles causes inhomogeneity in the local magnetic field, which leads to reduction of relaxation times of adjacent protons. Thus, the accumulation of iron oxide particles appears as dark (hypointense) regions on commonly used $T_{2}{ }^{*} / T_{2}$-weighted magnetic resonance images. ${ }^{5}$ The degree of hypointense image contrast, or the susceptibility effect, is highly dependent on relaxivity values and the iron
Correspondence: Nicola R Sibson Cancer Research UK and Medical Research Council Oxford Institute for Radiation Oncology, Department of Oncology, Old Road Campus Research Building, University of Oxford, Oxford OX3 7LJ, UK

Tel +44 I865225836

Fax +44 I86 585 7I 27

Email nicola.sibson@oncology.ox.ac.uk
International Journal of Nanomedicine 2018:13 4345-4359

4345

Dovepress f $y$ in 0

http://dx.doi.org/10.21474INN.S158071 (c) (i) The 2018 Zarghami et al. This work is published by Dove Medical Press Limited, and licensed under a Creative Commons Attribution License. and reproduction in any medium, provided the original author and source are credited. 
concentration of these agents, ${ }^{6,7}$ as well as MRI parameters such as magnetic field strength ${ }^{8,9}$ and pulse sequence. ${ }^{8,10,11}$ However, although $T_{2}$-weighted spin-echo (SE) and $T_{2}{ }^{*}$ weighted gradient-echo (GRE) sequences are regarded as the gold standard for iron oxide-enhanced MRI, ${ }^{12-14}$ and $7.0 \mathrm{~T}$ is the most commonly used field strength preclinically, a robust comparison of other sequences and/or magnetic field strengths has yet to be demonstrated.

Iron oxide agents are usually composed of a superparamagnetic core and a shell of varying thickness and material. In general, these particles are classified into 3 categories based on their hydrodynamic size. Ultra-small superparamagnetic iron oxide (USPIO; 5-50 nm in diameter) particles are the smallest agents currently in use. ${ }^{7,15}$ These particles typically exhibit a long (several hours) blood circulation half-life and have been clinically used for perfusion mapping and enhanced functional MRI activation detection. ${ }^{16,17}$ USPIO have also shown potential for imaging brain tumors with an impaired blood-brain barrier (BBB) ${ }^{18-20}$ Superparamagnetic iron oxide (SPIO; 50-150 $\mathrm{nm}$ in diameter) particles are slightly larger in size, ${ }^{14,20,21}$ and have seen extensive clinical use for lymph node imaging in cancer patients. ${ }^{22,23}$ Finally, microparticles of iron oxide (MPIO; $\sim \mu^{2,24,25}$ ) have been used in a variety of preclinical molecular imaging, ${ }^{25-29}$ but until recently ${ }^{30}$ a clinically compatible MPIO platform has not existed. Innovations and developments in the field of molecular imaging and biodegradable iron oxide contrast agents have increased the potential for translation of preclinical research to clinic..$^{30-32}$ However, selection of the appropriate USPIO, SPIO, or MPIO contrast agent for imaging depends largely on the application and research or clinical question to be addressed.

Over the last decade, molecular MRI, of brain-immune system interactions, has been the subject of numerous studies, ${ }^{27,33-38}$ with iron oxide particles being conjugated to a variety of different ligands (including proteins, peptides, antibodies, and carbohydrates) to target specific molecular epitopes expressed in disease. MPIO are often the agent of choice for such targeted molecular imaging studies, having several postulated advantages over UPSIO. The micron size of these particles enables assessment of specific binding to the endovasculature, unlike USPIO that are susceptible to macrophage uptake and passive diffusion into the brain parenchyma across a leaky BBB. ${ }^{39,40}$ Moreover, the iron content of a single MPIO is 4.3 million times higher than that of a USPIO, yielding considerably greater contrast per particle, with the contrast effect extending up to 50 times the physical size of the particle. ${ }^{6,24,41,42}$ Finally, the increased surface area of MPIO greatly increases labeling valency and, hence, binding affinity to the molecule of interest, while their short blood half-life ( $<5 \mathrm{~min}$ ) compared to USPIO yields a high target-to-background ratio. ${ }^{38,43,44}$ However, many of these advantages remain theoretical or have only been demonstrated in vitro, and a direct head-to-head in vivo comparison of molecularly targeted MPIO and USPIO has not previously been reported. ${ }^{37,39}$

The primary aims of the current study, therefore, were to 1) assess the relative sensitivities of 3 commonly used MRI sequences (single gradient echo 3D, multi-gradient echo 3D, and balanced steady-state free precession 3D) for the detection of iron oxide particles in mouse brain; 2) determine the effect of magnetic field strength on the detection of iron oxide particles in vivo; and 3) compare the specificity and sensitivity of vascular cell adhesion molecule (VCAM)MPIO and VCAM-USPIO for the detection of VCAM-1 in a mouse model of neuroinflammation.

\section{Materials and methods VCAM-MPIO synthesis}

Pro-mag ${ }^{\mathrm{TM}}$ carboxylic acid MPIO ${ }^{45}$ (Cat. No. PMC1N, Lot 10892, Bangs Laboratory Inc., Fishers, IN, USA) were vortexed and $1 \mathrm{mg}$ of MPIO transferred to a $2 \mathrm{~mL}$ plastic tube. 2-(N-morpholino)ethanesulfonic acid (MES) buffer was added (15 mM, $1 \mathrm{~mL}, \mathrm{pH}: 6.0)$. MPIO were pelleted on a Dynal magnetic separator (Thermo Fisher Scientific, Waltham, MA, USA) for $2 \mathrm{~min}$, and the supernatant discarded. For the amine coupling reaction, $100 \mu \mathrm{L}$ MES buffer and $100 \mu \mathrm{L}$ of a $10 \mathrm{mg} / \mathrm{mL}$ solution of 1-Ethyl-3-(3-dimethylaminopropyl)carbodiimide (EDC) (Cat. No. E6383, Sigma-Aldrich, St Louis, MO, USA) in $15 \mathrm{mM}$ MES pH 6.0 were added to the pelleted MPIO, and the solution was incubated for $30 \mathrm{~min}$ at $25^{\circ} \mathrm{C}$ on a shaker (1,000 rpm). The solution was washed twice with $1 \mathrm{~mL}$ of $15 \mathrm{mM}$ MES buffer and incubated with low endotoxin, $80 \mu \mathrm{L}$ azide-free monoclonal rat antibody to mouse VCAM-1 (clone M/K-2, Cat. No. 1510-14, SouthernBiotech, Birmingham, AL, USA) for $24 \mathrm{~h}$ at $25^{\circ} \mathrm{C}$ on a shaker. Conjugates were washed twice with PBS $/ 0.01 \%$ Tween and the supernatant containing any unbound antibody discarded. PBS was added to achieve the desired concentration of VCAM-MPIO.

\section{VCAM-USPIO synthesis}

Monocrystalline iron oxide nanocompounds (MION) were synthesized according to the published protocol. ${ }^{46}$ Physicochemical properties of MION have been well investigated previously. ${ }^{47} \mathrm{MION}$ were converted into cross-linked iron oxide (CLIO)- $\mathrm{NH}_{2}$ by reaction with epichlorohydrin and ammonium hydroxide. ${ }^{48} \mathrm{CLIO}-\mathrm{NH}_{2}$ were transformed into $\mathrm{CLIO}-\mathrm{COOH}$ by reaction with succinic anhydride. ${ }^{30}$ For VCAM-USPIO synthesis, $\operatorname{MES}(0.2 \mathrm{M}, 100 \mu \mathrm{L}, \mathrm{pH}: 6.0)$ was added to the solution 
of CLIO-COOH (1 mg iron, $100 \mu \mathrm{L}, 28.1 \mathrm{~nm}$ hydrodynamic diameter, $1.75 \times 10^{14}$ particles $/ \mathrm{mg}$ iron) and vortexed. Then, $10 \mu \mathrm{L}$ of N-hydroxysulfosuccinimide sodium salt (sulfoNHS) (10 $\mu \mathrm{mol}$; Cat. No. 56485, Sigma Aldrich) in MES buffer (1 M, pH 6.0) and $20 \mu \mathrm{L}$ of EDC solution (10 $\mu \mathrm{mol}$; Cat. No. E6383, Sigma Aldrich) in water were added. The mixture was incubated for $15 \mathrm{~min}$ at $25^{\circ} \mathrm{C}$ on a shaker (1,000 rpm), and excess of EDC was quenched by addition of $50 \mathrm{mg}$ of HL-COOH (Tentagel, Cat. No. 86.333, Sigma-Aldrich) for $15 \mathrm{~min}$. The sample was filtered and Tentagel was washed with $100 \mu \mathrm{L}$ of MES buffer (0.2 M, pH: 6.0). To this solution, $80 \mu \mathrm{L}$ of anti-mouse VCAM-1 antibody (same as MPIO) was added, and the reaction was incubated for $24 \mathrm{~h}$ at $25^{\circ} \mathrm{C}$ on a shaker. Subsequently, to quench any remaining activated carboxylic acid, $100 \mu \mathrm{L}$ of borate buffer (1M, $\mathrm{pH}$ : 8.5) was added and the solution incubated for another $24 \mathrm{~h}$ at $25^{\circ} \mathrm{C}$ on a shaker. The sample was centrifuged at $186,000 \mathrm{~g}$ for $1.5 \mathrm{~h}$, the supernatant discarded, and the pellet reconstituted in $1 \mathrm{~mL}$ of PBS. The sample was pelleted again $(186,000 \mathrm{~g}$ for $1.5 \mathrm{~h}$ ), then reconstituted in $1 \mathrm{~mL}$ of PBS and pelleted as before. This final pellet was reconstituted in $100 \mu \mathrm{L}$ of PBS.

\section{Physicochemical properties of VCAM-MPIO and VCAM-USPIO}

The particle size of VCAM-USPIO was measured by dynamic light scattering on a Zetasizer Nano ZS (Malvern Panalytical Ltd, Malvern, UK), and reported as the $z$-average diameter. The polydispersity is reported as the coefficient of variation $(\mathrm{CV})$ of the size distribution, which was calculated as the square root of the mean of the polydispersity index (for USPIO) or provided by the manufacturer (for MPIO). The zeta potential was measured on a Zetasizer Nano ZS in 0.1 M 4-(2-hydroxyethyl)piperazine-1-ethanesulfonic acid buffer at $\mathrm{pH}$ 7.0. The particle size of MPIO was provided by the manufacturer as a volume-weighted mean diameter measured with a Coulter counter. The iron content of VCAM-MPIO and VCAM-USPIO was measured by inductively coupled plasma optical emission spectrometry (ICP-OES) on a Varian Vista MPX (Varian, Palo Alto, CA, USA) after acid digestion. The upper limit of $\mathrm{COOH}$ loading was estimated from the nitrogen content of a freeze-dried sample, which was measured by combustion analysis. Results are reported as mean $\pm \mathrm{SD}$ of repeated measurements on the same sample.

\section{Iron content per particle and estimation of per-particle properties}

The mass of iron per particle was used as a conversion factor to relate intrinsic properties relative to the amount of iron (value per mmol of iron) to per particle properties (value for one particle). The mass of iron per particle was calculated as

$$
\text { pg Fe/particle }=10^{9} \times \frac{\% \mathrm{Fe}}{100} \times(\text { particles } / \mathrm{mg})^{-1}
$$

where $\% \mathrm{Fe}$ is the weight/weight concentration of iron in a dry sample measured by ICP-OES and particles/mg is the number of particles per mg of dry sample. The latter is provided by the manufacturer for MPIO, while for USPIO it is calculated as

$$
\begin{aligned}
& \text { Particles/mg } \\
& \quad=10^{-3} \times\left[\frac{4}{3} \pi\left(\frac{d}{2}\right)^{3}\left(\phi_{\mathrm{Fe}_{3} \mathrm{O}_{4}} \rho_{\mathrm{Fe}_{3} \mathrm{O}_{4}}+\left(1-\phi_{\mathrm{Fe}_{3} \mathrm{O}_{4}}\right) \rho_{\mathrm{Dex}}\right)\right]^{-1}
\end{aligned}
$$

where $d$ is the particle diameter, $\phi_{\mathrm{Fe}_{3} \mathrm{O}_{4}}$ is the volume fraction of magnetite in a dry sample, $\rho_{\mathrm{Fe}_{3} \mathrm{O}_{4}}$ is the density of magnetite, and $\rho_{\text {Dex }}=1.07 \mathrm{~g} / \mathrm{cm}^{3}$ is the density of hydrated cross-linked dextran. ${ }^{49}$ The volume fraction of magnetite was calculated from the mass fraction of magnetite $\chi_{\mathrm{Fe}_{3} \mathrm{O}_{4}}=(\% \mathrm{Fe} / 100) \times\left[\left(3 A_{r}(\mathrm{Fe})+4 A_{r}(\mathrm{O})\right) /\left(3 A_{r}(\mathrm{Fe})\right)\right]$, where $A_{r}(\mathrm{Fe})=55.845 \mathrm{u}$ and $A_{r}(\mathrm{O})=15.999 \mathrm{u}$ are the atomic weights of iron and oxygen, respectively, and the density ratio $\rho_{\mathrm{Fe}_{3} \mathrm{O}_{4}} / \rho_{\text {Dex }}$

\section{Assessment of VCAM antibody loading on MPIO and USPIO}

MPIO $(10 \mu \mathrm{g})$ or USPIO $(70 \mu \mathrm{g})$ were diluted in 200 of PBS buffer, and $2 \mu \mathrm{L}$ of Alexa Fluor 647 goat anti-rat $\operatorname{IgG}(\mathrm{H}+\mathrm{L})$ (Cat. No. A21091, 5 fluorophores per antibody, Invitrogen) was added. For MPIO, samples were shaken for $30 \mathrm{~min}$, pelleted using a magnet, washed twice with PBS/0.01\% Tween, and resuspended in $400 \mu \mathrm{L}$ of PBS. Qifikit calibration beads (Dako, Agilent Technologies, Stockport, UK), used as a reference, were prepared according to the manufacturer's protocol, but substituting the provided fluorescently-conjugated antibody by Alexa Fluor 647 goat anti-mouse IgG $(\mathrm{H}+\mathrm{L})$ (Cat. No. A21050, 5 fluorophores per antibody, Invitrogen). Flow cytometry experiments were performed on a BD FACSCalibur flow cytometer, using channel FL4 (661/16 nm band pass filter). Calibration curve was performed, according to manufacturer protocol, by linear regression of the $\log -\log$ plot of antibody loading versus fluorescence intensity. For USPIO, sample was centrifuged at $186,000 \mathrm{~g}$ for $1.5 \mathrm{~h}$, the supernatant discarded, and the pellet reconstituted in $1 \mathrm{~mL}$ of PBST. The sample was pelleted again $(186,000 \mathrm{~g}$ for $1.5 \mathrm{~h})$ and reconstituted in $200 \mu \mathrm{L}$ of PBS. Fluorescence intensity 
of the samples was measured and compared to a calibration curve composed of serial dilutions of Alexa Fluor 647 goat anti-rat $\operatorname{IgG}(\mathrm{H}+\mathrm{L})$. Results are reported as mean $\pm \mathrm{SD}$.

\section{Determination of MPIO and USPIO relaxivity in agarose gel}

MRI phantoms were prepared as described previously. ${ }^{30}$ Unconjugated MPIO and USPIO were embedded in $2 \%$ $\mathrm{w} / \mathrm{v}$ agarose gels at iron concentrations of $0.0025-0.2 \mathrm{mM}$. Gels were prepared in $15 \mathrm{~mL}$ tubes and heated at $95^{\circ} \mathrm{C}$. MPIO and USPIO particles were added to the tubes and dispersed for a homogenous mixture. Samples were sonicated and kept undisturbed at room temperature until cool. MPIO and USPIO relaxitivities were measured using a SE multi-slice sequence in a 7.0 T horizontal bore system (Agilent Technologies, Santa Clara, CA, USA) with a 26-mm birdcage RF coil (Rapid Biomedical, Rimpar, Germany). Sequence parameters for the $T_{2}$ maps were: repetition time $(\mathrm{TR})=10 \mathrm{~s}, 12 \times$ echo time $(\mathrm{TE})=[10-300] \mathrm{ms}$, spectral width $(\mathrm{SW})=100 \mathrm{kHz}$, matrix $=128 \times 128$, field of view $(\mathrm{FoV})=72 \times 72 \mathrm{~mm}$, slice thickness $=2 \mathrm{~mm}$, and experimental time $($ Texp $)=4 \mathrm{~h} 16 \mathrm{~min}$. Sequence parameters for the $T_{1}$ maps were: $\mathrm{TR}=10 \mathrm{~s}, \mathrm{TE}=10 \mathrm{~ms}, 12 \times \mathrm{Ti}=[0.01-6.0] \mathrm{s}$, $\mathrm{SW}=100 \mathrm{kHz}$, matrix $=128 \times 128, \mathrm{FoV}=72 \times 72 \mathrm{~mm}$, and slice thickness $=2 \mathrm{~mm}$, Texp $=4 \mathrm{~h} 16 \mathrm{~min}$.

\section{Assessment of VCAM-MPIO and VCAM-USPIO circulatory half-life}

To determine the blood half-life of VCAM-MPIO and VCAM-USPIO, the particles were injected via a tail vein into female BALB/c mice (6-7 weeks old, $\mathrm{n}=15$, Charles River, $\mathrm{UK}, \mathrm{Kent}, \mathrm{UK}$ ) at a constant dose of $4 \mathrm{mg} \mathrm{Fe} / \mathrm{kg}$ in $100 \mu \mathrm{L}$ sterile PBS. At $0,0.8,1,2,2.5,3,5$, and 10 min after injection for VCAM-MPIO and 1, 6, 13, 24, 48 and 72, $96 \mathrm{~h}$ after VCAM-USPIO injection, the mice underwent anesthesia with $2 \%-3 \%$ isoflurane in $30 \% \mathrm{O}_{2}$ with $70 \% \mathrm{~N}_{2}$ followed by thoracotomy. Blood was collected from the left ventricle using a $1 \mathrm{~mL}$ syringe with a $23 \mathrm{G}$ needle containing $1 \mu \mathrm{L}$ of heparin sodium (25,000 I.U./mL, Wockhardt Ltd, Wrexham, UK). Blood samples (up to $1 \mathrm{~mL}$ ) were oxygenated by placing in $15 \mathrm{~mL}$ tubes with a $100 \% \mathrm{O}_{2}$ atmosphere and inverting gently for 5 min until the color changed to bright red. Final blood volume $(100 \mu \mathrm{L})$ was mixed with $1 \%$ agarose solution in PBS $(100 \mu \mathrm{L})$, at $41{ }^{\circ} \mathrm{C}$ and placed in $500 \mu \mathrm{L}$ microcentrifuge tube. $T_{2}$ relaxivities of samples were immediately measured using a 7.0 T MRI. For $T_{2}$ relaxometery, a simple $90^{\circ}$ and $180^{\circ}$ pulse sequence were used with long TR (10 s) and adjusted array of TE for best fitting. Circulatory blood half-life was determined from the fitted 1 phase decay curve, constrained to a plateau at the average naive blood sample value (baseline). Blood samples from naïve mice were used as the baseline $(n=6)$.

\section{Mouse neuroinflammation model}

All animal procedures were performed under the University of Oxford guidelines and approved by the UK Home Office. Female BALB/c mice (6-7 weeks old, $\mathrm{n}=24$; Charles River, Kent, UK) were anesthetized with isoflurane (1.5\%-2.0\%) in $30 \% \mathrm{O}_{2}: 70 \% \mathrm{~N}_{2} \mathrm{O}$, placed in a stereotaxic frame and a burr hole drilled above the injection site (co-ordinates from bregma: anterior $+0.5 \mathrm{~mm}$; left $2.0 \mathrm{~mm}$; depth $2.5 \mathrm{~mm}$ ). Using a glass microcannula (tip diameter $c a .<50 \mu \mathrm{m}), 1 \mathrm{ng}$ of mouse recombinant interleukin-1 $\beta$ (IL-1 $\beta$, Peprotech EC, London, UK) in $1 \mu \mathrm{L}$ sterile PBS was injected into the left striatum to induce unilateral vascular activation. ${ }^{25}$ The scalp incision was sutured, and the animals were recovered from anesthesia. Three hours after the surgery, ${ }^{25}$ the mice were injected via tail vein with the dose of $4 \mathrm{mg} \mathrm{Fe} / \mathrm{kg}$ of either (a) VCAM-MPIO ( $n=12)$ or (b) VCAM-USPIO $(n=12)$.

\section{MRI}

All MRI experiments were performed using horizontal, wide-bore superconductive 4.7, 7.0, or 9.4 T MRI systems (Agilent Technologies Inc). The same size (ID =26 mm) transmit/receive birdcage RF coils were used in all experiments (Rapid Biomedical).

Mice injected intravenously with VCAM-MPIO ( $n=4$ for each field strength) were imaged $1 \mathrm{~h}$ after contrast agent administration, as previously reported. An imaging timepoint of $13 \mathrm{~h}$ post-injection was chosen for mice injected with VCAM-USPIO ( $\mathrm{n}=4$ for each field strength) to account for the longer circulation half-life. ${ }^{50,51}$ All mice were imaged with both multi-gradient echo and balanced steady-state free precession 3D sequences (MGE3D and bSSFP3D, respectively). For MGE3D, the parameters were as follows: excitation angle $=15^{\circ} ; \mathrm{TR}=65.1 \mathrm{~ms}$; time of the $1 \mathrm{st}$ echo $\left(\mathrm{TE}_{1}\right)=2.5 \mathrm{~ms}$; echo separation time $\left(\mathrm{TE}_{2}\right)=4 \mathrm{~ms}$ (even echoes acquired in the reverse k-space direction, during opposite read gradient); $\mathrm{SW}=100 \mathrm{kHz}$ (acquisition time $=2.56 \mathrm{~ms}$ ); and number of echoes $=15$. For bSSFP3D, the parameters were as follows: excitation angle $=20^{\circ}$; TR $/ \mathrm{TE}=8 / 4 \mathrm{~ms} ; \mathrm{SW}=147 \mathrm{kHz}$ (acquisition time $=1.74 \mathrm{~ms}$ ); and number of excitation frequency offsets $=8$. The following parameters were consistent for both sequences: $\mathrm{FoV}=22.5 \times 22.5 \times 22.5 \mathrm{~mm}^{3}$; acquisition matrix size $=256 \times 192 \times 192$ (final images zero-filled to 
$256 \times 256 \times 256$; final isotropic resolution $=88 \mu \mathrm{m})$. Both MGE3D and bSSFP3D scans were run with a single average $(\mathrm{NT}=1)$, and the total acquisition time was $\sim 40 \mathrm{~min}$ for each.

\section{Image reconstruction}

Images were reconstructed using home-built MATLAB (MathWorks, Natick, MA, USA) code. The final MGE3D images were reconstructed as the square root of sum of squares (SqrtSOS) of individual echoes. ${ }^{52-54}$ Based on previous data, second single echo $(\mathrm{TE}=6.5 \mathrm{~ms})$ was extracted from the MGE3D sequence to represent a single echo gradient echo (GE3D) dataset. ${ }^{33,55}$ For bSSFP3D, the final images were reconstructed by adding the individual frequency offsets using the same SqrtSOS algorithm as above. ${ }^{56,57}$

\section{Image and data analysis}

For all datasets, brain masks were generated manually. Quantification of hypointense signals from MPIO and USPIO was performed using in-house MATLAB code. Signals arising from ventricles or sinuses, which naturally appear hypointense, were excluded. ${ }^{27}$ The quantification of signal-to-noise (SNR) was performed by measuring the signal intensity $\left(\mathrm{SI}_{\text {brain }}\right)$ within the mouse brain (excluding hypointensities) and $\mathrm{SD}$ of background noise $\left(\mathrm{SD}_{\text {noise }}\right)$. For contrast-to-noise (CNR), we additionally measured the signal intensity coming from VCAM-MPIO or VCAM-USPIO ( $\left.\mathrm{SI}_{\text {void }}\right)$. Thus, SNR and CNR were estimated using the following calculations, respectively:

$$
\begin{gathered}
\mathrm{SNR}=\frac{\mathrm{SI}_{\text {brain }}}{\mathrm{SD}_{\text {noise }}} \\
\mathrm{CNR}=\frac{\mathrm{SI}_{\text {brain }}-\mathrm{SI}_{\text {void }}}{\mathrm{SD}_{\text {noise }}}
\end{gathered}
$$

Statistical analyses were performed using Prism software (GraphPad, San Diego, CA, USA). The normality of the measured variables was tested using the Shapiro-Wilk test. For multiple comparisons, analysis of variance (ANOVA) followed by Tukey post-hoc test were used.

\section{Ex vivo assessment of VCAM-MPIO and USPIO binding}

Following imaging, mice were transcardially perfused under terminal anesthesia with $0.9 \%$ heparinized saline followed by periodate-lysine-paraformaldehyde (PLP-light) containing $0.1 \%$ glutaraldehyde. Brains were harvested, post-fixed in PLP then placed in $30 \%$ sucrose solution until they sank to the bottom. Samples were frozen in isopentane at $-80^{\circ} \mathrm{C}$. Brains were cut at $10 \mu \mathrm{m}$ and kept at $-20^{\circ} \mathrm{C}$ until immunohistochemical staining for co-localization of VCAM-1 and iron oxide particles. Sections were quenched with $1 \%(\mathrm{v} / \mathrm{v})$ hydrogen peroxide $(30 \% \mathrm{w} / \mathrm{v})$ in methanol (Sigma Aldrich), blocked with $10 \%$ goat serum in PBS for $1 \mathrm{~h}$, and then incubated overnight at $4^{\circ} \mathrm{C}$ with the primary VCAM-1 antibody (1:100; SouthernBiotech). Sections were washed using PBS/0.01\% Tween (Sigma Aldrich), and then incubated with a biotinylated rat polyclonal secondary antibody (1:200 Abcam, Cambridge, UK) for $1 \mathrm{~h}$. Slides were washed and then incubated in Vectorelite ABC kit (1:1:100; Vector Laboratories, Peterborough, UK) for $45 \mathrm{~min}$. The peroxidase was visualized using 3,3'diaminobenzidine (Sigma Aldrich). To detect the iron oxide particles, sections were stained using Perls' Prussian blue. Positive control liver and spleen samples from mice injected intravenously with USPIO were included for Perls' Prussian blue staining. For fluorescent VCAM-1 staining, Tris- $\mathrm{NaCl}$ blocking buffer (TNB; PerkinElmer, Seer Green, UK) was used. Sections were subsequently incubated overnight at $4^{\circ} \mathrm{C}$ with the VCAM-1 primary antibody, as above. Sections were washed with PBS and incubated with biotinylated anti-goat secondary in TNB for $30 \mathrm{~min}$. Sections were then washed with PBS, incubated with streptavidin-horseradish peroxidase (1:200; PerkinElmer) in TNB for $30 \mathrm{~min}$, washed and incubated for $7 \mathrm{~min}$ in the dark with tyramide signal amplification (TSA)-biotin (1:100) in amplification buffer (PerkinElmer). Slides were washed and incubated with a streptavidin-DyLight 488 (1:200; Vector Laboratories). Samples were coverslipped, using Vectashield mounting medium containing DAPI(Vector Laboratories). Whole brain fluorescent images were taken at $20 \times$ magnification using an Aperio immunofluorescence slide scanner (Leica Biosystems, Milton Keynes, UK).

\section{Results}

\section{Physicochemical properties of VCAM-MPIO and VCAM-USPIO}

The $z$-average hydrodynamic diameter of VCAM-USPIO was $28.1 \mathrm{~nm}$. The particle size distribution (Figure S1) is moderately polydisperse, with a $\mathrm{CV}$ of $36 \%$. The particle size distribution of MPIO was narrower, with a CV of $15 \%$ and a mean diameter of $880 \mathrm{~nm}$. Both USPIO and MPIO were negatively charged, with a zeta potential of $-9.3 \pm 0.9 \mathrm{mV}$ and $-25.6 \pm 1.3 \mathrm{mV}$, respectively. The iron content of USPIO and MPIO was $30.6 \% \mathrm{w} / \mathrm{w}$ and $27.8 \% \mathrm{w} / \mathrm{w}$, respectively, as measured by ICP-OES. 
Quantitation of antibody loading showed the density of VCAM antibody to be $10,490 \pm 450$ molecules per particle $(n=4)$ on MPIO and 7.4 \pm 3.1 molecules per particle on USPIO $(n=3)$. Although the number of antibodies per particle differs by 3 orders of magnitude, the surface density (number of antibodies per unit area) is comparable, with $4,320 \pm 200$ molecules $/ \mu^{2}$

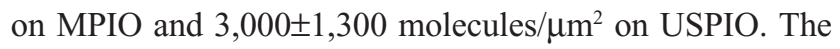
zeta potential of either agents does not change significantly after conjugation to the antibody $(-10.3 \pm 1.1 \mathrm{mV}$ for VCAMUSPIO and $-26.4 \pm 1.4 \mathrm{mV}$ for VCAM-MPIO), which is not entirely unexpected as the measurement $\mathrm{pH}$ falls within the isoelectric point range of rat $\mathrm{IgG} .^{58}$

The $R_{2}$ relaxivity of MPIO and USPIO, measured in agarose phantoms, was found to be $500 \mathrm{~Hz} \mathrm{~L} / \mathrm{mmol} \mathrm{Fe}$ and $126 \mathrm{~Hz} \mathrm{~L} / \mathrm{mmol}$ Fe, respectively (Figure S2A and B). Both values are consistent with previous literature reports ${ }^{30,37,59,60}$ and correspond to average relaxivitiy per particle of $1.18 \times 10^{-9} \mathrm{~Hz} \mathrm{~L} /$ particle for MPIO and the much lower value of $1.29 \times 10^{-14} \mathrm{~Hz} \mathrm{~L} /$ particle for USPIO. Properties of MPIO and USPIO are summarized in Table 1.

\section{Comparison of MRI sequences and field strengths for detection of iron oxide particles}

Representative images obtained using each of the 3 sequences (MGE3D, GE3D, and bSSFP3D) at 4.7, 7.0, and 9.4 T, with the same resolution and scan time, are shown in Figures 1A and 2A. Qualitatively, all 3 sequences enabled visualization of hypointense foci in the brains of mice injected with either

Table I Physicochemical properties of MPIO and USPIO

\begin{tabular}{|c|c|c|}
\hline Properties & MPIO & USPIO \\
\hline Mean diameter $(\mathrm{nm})$ & $880^{a}$ & 28.1 \\
\hline Coefficient of variation ${ }^{\mathrm{b}}(\%)$ & $15^{\mathrm{a}}$ & 36 \\
\hline Iron content $(\% \mathrm{w} / \mathrm{w})$ & 27.8 & 30.6 \\
\hline Core diameter $(\mathrm{nm})$ & $5.1^{c}$ & $9-14^{d}$ \\
\hline Saturation magnetization (emu/g Fe) & $115^{c}$ & $62^{\mathrm{e}}$ \\
\hline Zeta potential $(\mathrm{mV})$ & $-26.4 \pm 1.4^{f}$ & $-10.3 \pm 1.1^{g}$ \\
\hline $\mathrm{COOH}$ loading ( $\mu \mathrm{mol} / \mathrm{mg} \mathrm{Fe})$ & $122^{\mathrm{a}}$ & $<1.24$ \\
\hline Surface area of I particle $\left(\mu \mathrm{m}^{2}\right)$ & 2.4 & 0.0025 \\
\hline Iron content per particle (pg Fe/particle) & 0.13 & $5.7 \times 10^{-6}$ \\
\hline Antibody density per particle (Ab/particle) & $10,490 \pm 450^{g}$ & $7.4 \pm 3.1^{18}$ \\
\hline Antibody density per unit area $\left(\mathrm{Ab} / \mu \mathrm{m}^{2}\right)$ & $4,320 \pm 200^{g}$ & $3,000 \pm 1,300 \mathrm{~g}$ \\
\hline Bulk relaxivity $(\mathrm{Hz} \mathrm{L} / \mathrm{mmol} \mathrm{Fe})$ & 500 & 126 \\
\hline Particle relaxivity (Hz L/particle) & $1.18 \times 10^{-9}$ & $1.29 \times 10^{-14}$ \\
\hline
\end{tabular}

Notes: a Data provided by the manufacturer. ${ }^{b}$ Relative SD of particle diameters within a sample. 'From the study by Delangre et al. ${ }^{59}{ }^{\mathrm{d}}$ From the study by PerezBalderas et al. ${ }^{30}$ eFrom the study by Chen et al. ${ }^{58}{ }^{\mathrm{f}}$ Calculated from diameter assuming spherical particles. ${ }^{8}$ Data are reported as mean \pm SD of repeated measurements of the same sample.

Abbreviations: MPIO, microparticles of iron oxide; USPIO, ultra-small superparamagnetic iron oxide.
VCAM-MPIO (Figure 1A) or VCAM-USPIO (Figure 2A). However, the $T_{2}{ }^{*}$-weighted MGE3D sequence appeared to reveal hypointensities more prominently than either the $T_{2}{ }^{*}$ weighted GE3D or the $T_{2} / T_{1}$-weighted bSSFP3D sequence. Quantitative assessment of SNR (Figure 3A and C) and CNR (Figure 3B and D) for all sequences at each magnetic field confirmed that the highest SNR was conferred by the MGE3D sequence compared with both the other sequences for both MPIO and USPIO (in all cases, ANOVA $P<0.0001$, followed by Tukey's post-hoc test; Figure 3). The $T_{2} / T_{1}$-weighted bSSFP3D sequence also showed significantly greater CNR than the standard GE3D sequence for the USPIO (Figure 3D). When comparing SNR and CNR of the optimal MGE3D sequence at different magnetic field strengths, a significant increase in both SNR and CNR was evident from 4.7 to $7.0 \mathrm{~T}$ (in all cases, ANOVA $-P<0.05$, followed by Tukey's post-hoc test). However, no additional increase in either signal or contrast was gained by increasing field strength from 7.0 to $9.4 \mathrm{~T}$.

\section{Comparing sensitivity and specificity of VCAM-MPIO and VCAM-USPIO}

Immunohistochemically, unilateral upregulation of VCAM-1 was evident in mice injected intracerebrally with IL-1 $\beta$ and intravenously with VCAM-MPIO (Figure 1B-D). In accord with this unilateral upregulation, a marked unilateral contrast effect was evident in brain images at all field strengths and with all 3 sequences (Figure 1A). In contrast, in mice injected intracerebrally with IL- $1 \beta$ and intravenously with VCAMUSPIO, bilateral contrast effects were evident in all animals, with no differences between the hemispheres (Figure 2A). Immunohistochemistry confirmed unilateral VCAM-1 upregulation (Figure 2B-D). Quantitative analysis of hypointense voxels (mean $\pm \mathrm{SE}$ ) for all 3 field strengths showed a significant difference between the number of hypointense voxels in the injected and contralateral hemispheres for mice injected with VCAM-MPIO (4.7 T: $446 \pm 119$ vs $229 \pm 30$; 7.0 T: $430 \pm 47$ vs $253 \pm 47 ; 9.4 \mathrm{~T}: 425 \pm 98$ vs $214 \pm 58 ; \mathrm{n}=4$ per group, $P<0.05$; Figure $4 \mathrm{~A}$ ), but no significant difference for VCAM-USPIO-induced hypointensities ( $4.7 \mathrm{~T}: 1,444 \pm 75$ vs $1,213 \pm 125 ; 7.0$ T: $1,244 \pm 90$ vs $1,138 \pm 36$; 9.4 T: $1,058 \pm 28$ vs $933 \pm 95 ; n=4$ per group). Immunohistochemically, VCAM-1 and Perls' Prussian blue staining revealed the presence of VCAM-MPIO in the lumen of VCAM-1-positive vessels following MRI. However, despite intense bilateral contrast effects observed by MRI, no VCAM-USPIO were detected on VCAM-1 positive vessels or within the brain parenchyma following MRI (Figure 4B). To test that Perls' Prussian blue 
A
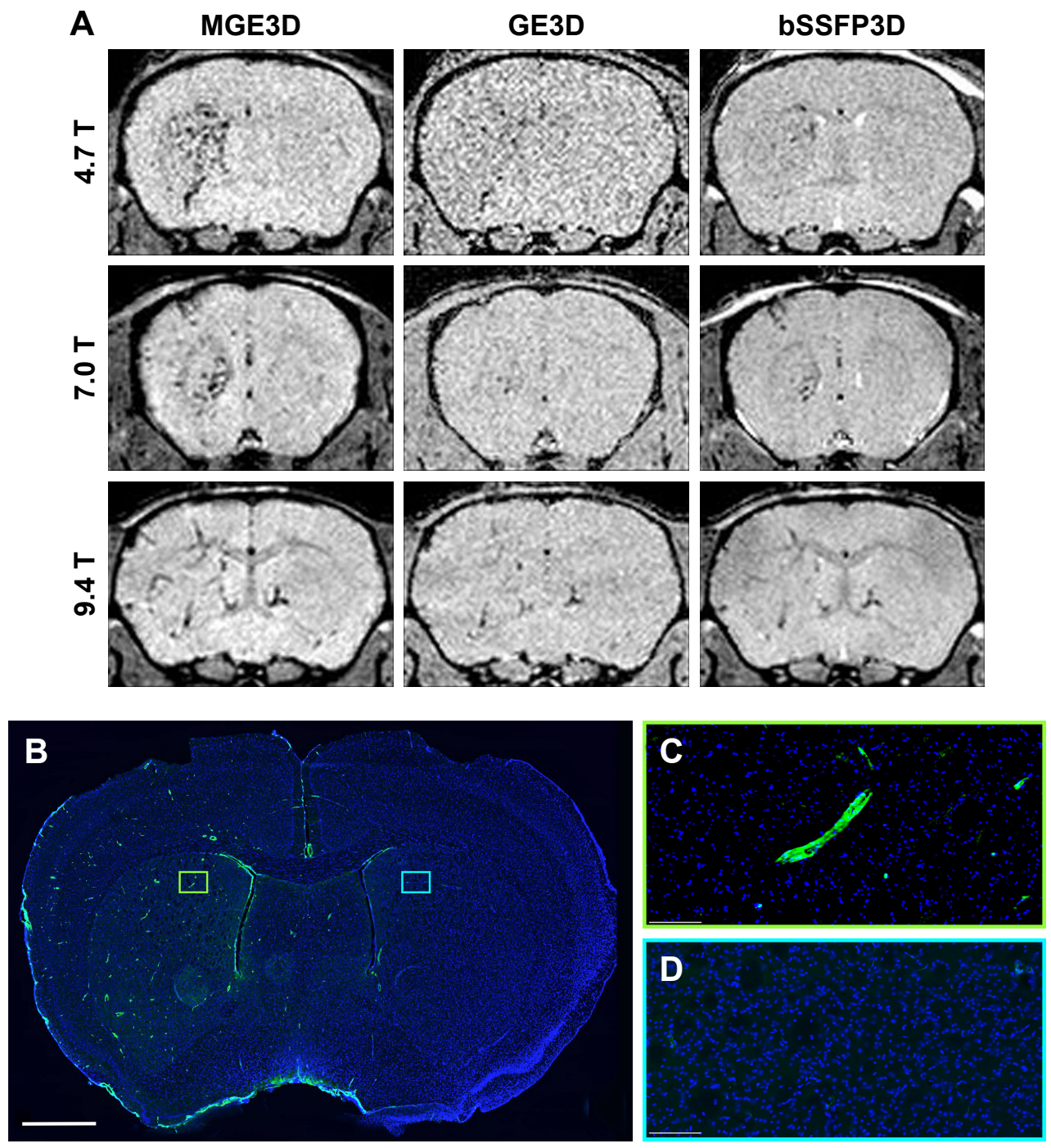

Figure I (A) Representative in vivo images of mice I h post-VCAM-MPIO injection at different magnetic fields. Three sequences of MGE3D, GE3D, and bSSFP3D are shown from a 3D dataset. Intense low signal areas on the left side of the brain reflect the specific retention of MPIO on acutely activated vascular endothelium with visually absent contrast effect in the contralateral control hemisphere. MGE3D demonstrates better visualization of hypointensities compared with other sequences. (B-D) Histological assessment of VCAM upregulation at the injection side.

Notes: Green vessels show unilateral upregulation of VCAM, nuclei are shown in blue. Scale bar: I mm.

Abbreviations: bSSFP3D, balanced steady-state free precession 3D; GE3D, gradient echo 3D; MGE3D, multi-gradient echo 3D; MPIO, microparticles of iron oxide; VCAM, vascular cell adhesion molecule.

stain can, in fact, stain and detect USPIO, positive control samples (liver and spleen) from mice injected with USPIO were stained. The results indicate that it is possible to detect USPIO (Figure S3) histologically. ${ }^{61}$

To determine whether contrast effects could be arising from unbound VCAM-USPIO within the blood, the half-life of VCAM-USPIO in the circulation was measured and found to be $16.5 \mathrm{~h}$, in comparison to $1.2 \mathrm{~min}$ for VCAM-MPIO (Figure 4C). Consequently, the bilateral contrast effects arising from VCAM-USPIO administration was further investigated in both naïve mice and mice injected intracerebrally with IL-1 $\beta$. Mice were injected intravenously with VCAMUSPIO and imaged at different time points $(1,13$, and $80 \mathrm{~h})$ after injection, as above (Figure 4D). Results showed bilateral contrast effects and, hence, presence of VCAM-USPIO, in both cohorts at 1 and $13 \mathrm{~h}$ after injection. In both naïve and IL- $1 \beta$ injected mice, contrast effects arising from VCAMUSPIO were no longer evident $80 \mathrm{~h}$ after injection (ie, $c a$. $5 \times$ blood half-life of VCAM-USPIO).

\section{Discussion}

In this study, we have demonstrated that the $T_{2}{ }^{*}$-weighted multi-gradient echo sequence provides the highest SNR and CNR for detection of targeted iron oxide particles in mouse brain. All sequences showed an improvement in both SNR and CNR on increasing field strength from 4.7 to $7.0 \mathrm{~T}$, but 

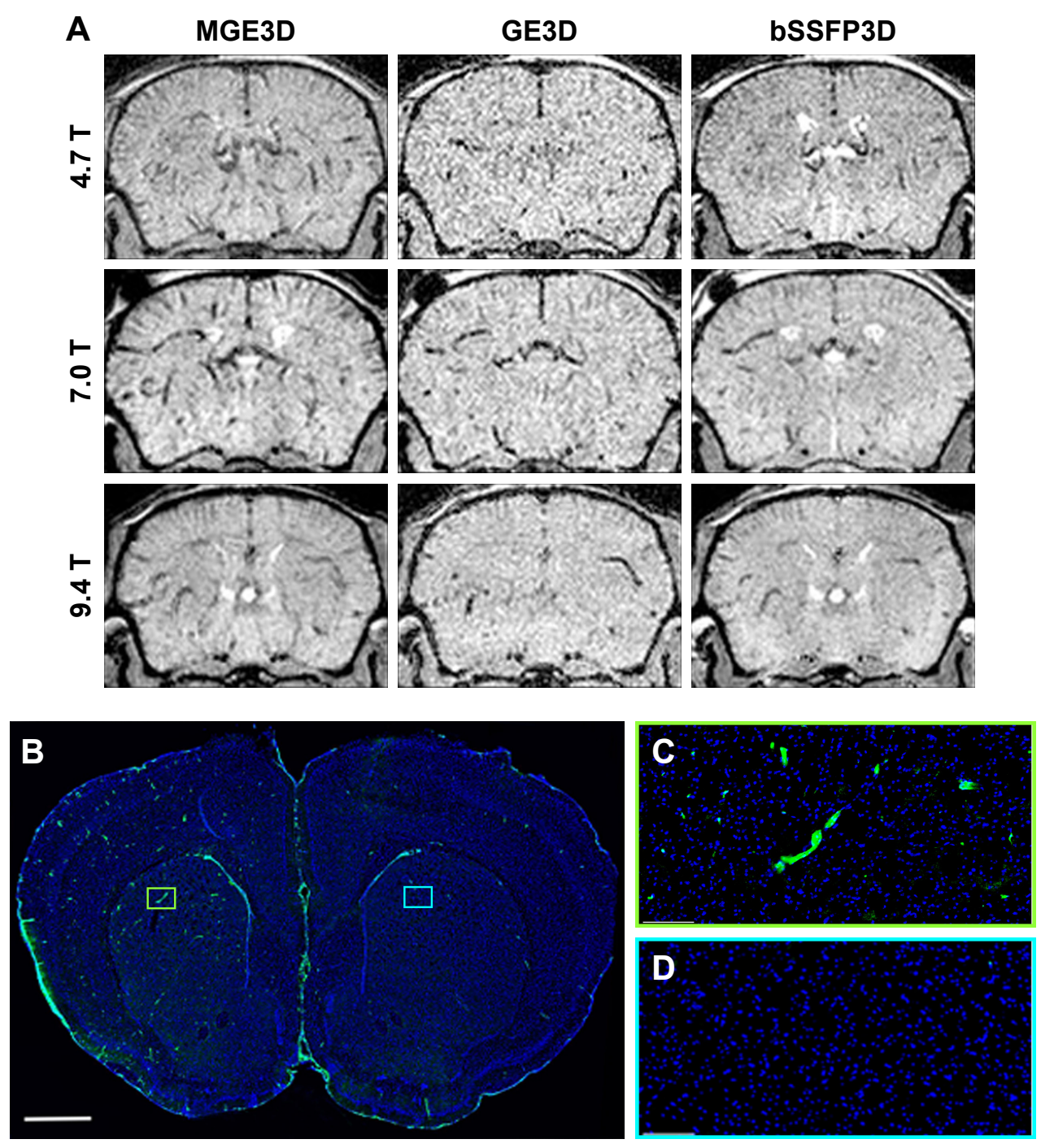

Figure 2 (A) Representative in vivo images of mice $13 \mathrm{~h}$ post-VCAM-USPIO injection at different magnetic fields. Three sequences of MGE3D, GE3D, and bSSFP3D are shown from a 3D dataset. Hypointensities were detected in both hemispheres of the brains. (B-D) Histological assessment of VCAM upregulation at the injection side. Notes: Green vessels show unilateral upregulation of VCAM, nuclei are shown in blue. Scale bar: I mm.

Abbreviations: bSSFP3D, balanced steady-state free precession 3D; GE3D, gradient echo 3D; MGE3D, multi-gradient echo 3D; USPIO, ultra-small superparamagnetic iron oxide; VCAM, vascular cell adhesion molecule.

no further improvements in these parameters were observed on going to higher field. Finally, we have shown that while targeted MPIO enable selective and specific detection of target expression on the cerebral vasculature, the long halflife and lower relaxivity, and hence CNR, per particle of the targeted USPIO will likely render these particles impractical for most applications of molecular MRI targeting endovascular ligands.

Several different MRI approaches have been used previously for mouse neuroimaging of iron oxide contrast effects. As the transverse magnetization decays with $T_{2}{ }^{*}$, it distorts the gradient echo and, therefore, induces geometrical distortions. ${ }^{62}$ Hence, conventional $T_{2}{ }^{*}$-weighted gradient echo (GE3D) is the most commonly used sequence for imaging of these particles. ${ }^{36}$ This sequence uses a single echo sampled per line in k-space. In contrast, for $T_{2}{ }^{*}$-weighted multi-echo gradient echo (MGE3D), the recovery time is used to acquire additional echoes without increasing the total experimental time. Thus, a series of echoes at different $\mathrm{TE}$ are recorded for each line in $\mathrm{k}$-space. This additional information can be used in a number of different ways, such as applying a SqrtSOS algorithm on the magnitude images from each echo to obtain MGE3D images. ${ }^{63}$ The bSSFP3D sequence also has been used previously for tracking cells labeled with iron oxide particles and has shown advantages over conventional $T_{2}$ - and $T_{2}{ }^{*}$-weighted sequences. ${ }^{29,41,64}$ 
A

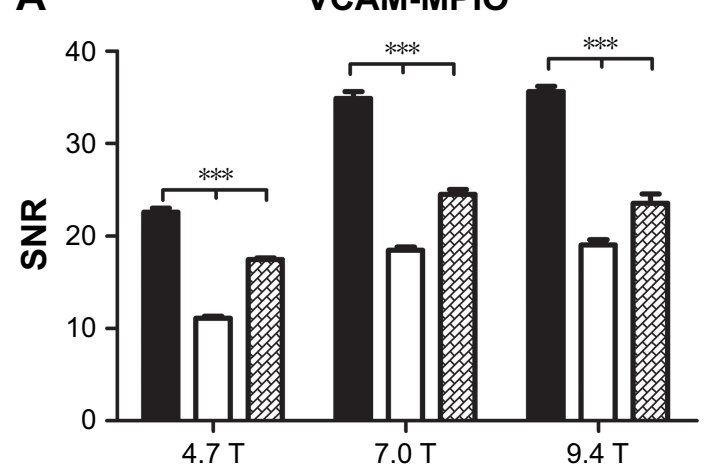

B

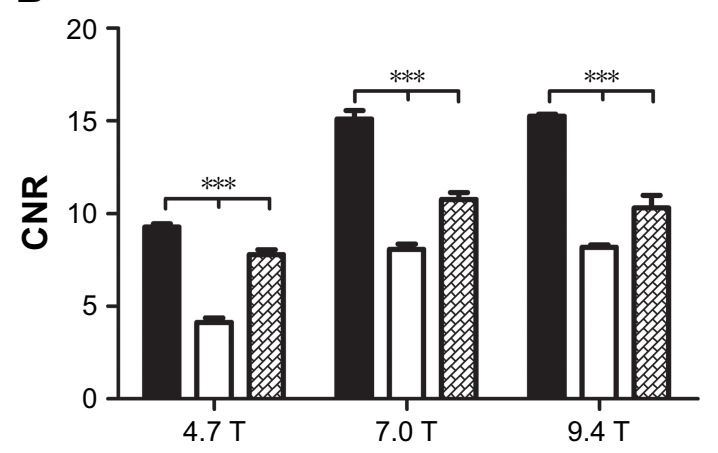

C

VCAM-USPIO

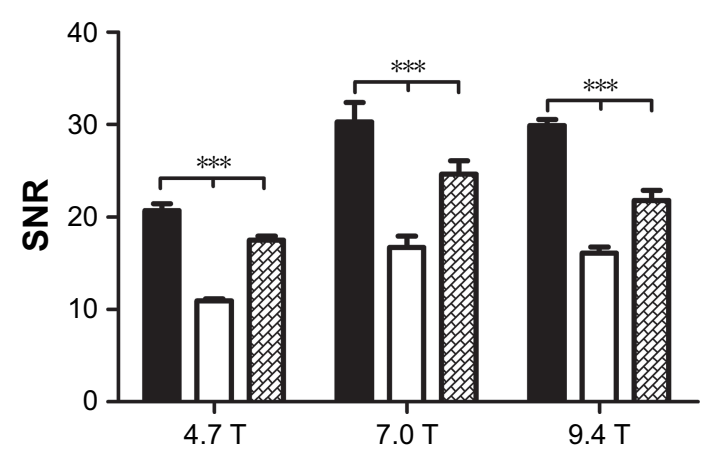

D

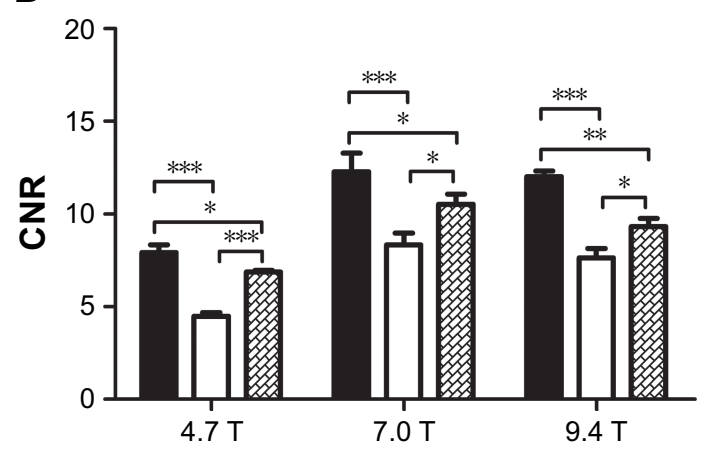

Figure 3 Quantitative comparison of MGE3D ( $\square$ ), GE3D ( $\square$ ), and bSSFP3D (四) sequences for detection of MPIO and USPIO at different magnetic field strengths. Note: MGE3D yielded significantly higher SNR $(\mathbf{A}, \mathbf{C})$ and CNR $(\mathbf{B}, \mathbf{D})$ compared with other sequences at all field strengths for both MPIO and USPIO detection $(* P<0.05$, $* * P<0.0$ I, $* * * P<0.00$ I).

Abbreviations: bSSFP3D, balanced steady-state free precession 3D; CNR, contrast-to-noise ratio; GE3D, gradient echo 3D; MGE3D, multi-gradient echo 3D; MPIO, microparticles of iron oxide; SNR, signal-to-noise ratio; USPIO, ultra-small superparamagnetic iron oxide; VCAM, vascular cell adhesion molecule.

However, this sequence is highly sensitive to local field inhomogeneities and the resulting "banding artifact" worsens at higher magnetic fields. ${ }^{65,66}$

The results of the current study suggest that by combining echoes in the MGE3D dataset, both SNR and CNR show significant improvements. Thus, MGE3D provides better depiction of both VCAM-MPIO and VCAM-USPIO contrast effects and greater overall image quality and sharpness compared to conventional GE3D and bSSFP3D. Consequently, quantitation of contrast effects will be improved significantly using the MGE3D sequence.

With regard to field strength, theoretically, an improvement in SNR would be anticipated with increasing field strength. However, many factors can limit the results observed in practice. In this study, improvements in both SNR and CNR were achieved for all sequences between 4.7 and 7.0/9.4 T, in accord with other rodent neuroimaging studies. ${ }^{67,68}$ Although a significant improvement was observed between 4.7 and 7.0 T, no significant gain was obtained by increasing field strength further to $9.4 \mathrm{~T}$ in the current study. One possible reason for this lack of further
SNR benefit is that magnetic susceptibility inherent in a mouse brain produce field-dependent perturbations in $\mathrm{B}_{0}$ homogeneity, which result in degradation of SNR at 9.4 T. However, direct comparison of different magnetic field strengths suffers from several practical limitations. ${ }^{67}$ For example, one consideration is the effect of different bore sizes. For the systems used in this study, the $9.4 \mathrm{~T}$ scanner had the smallest bore size (ID $160 \mathrm{~mm}$ ), only allowing the use of a gradient coil with lower performance (gradient coil dimensions: OD $156 \mathrm{~mm}$, ID $100 \mathrm{~mm}$ ) than the $210 \mathrm{~mm}$ bore 7.0 $\mathrm{T}$ and $310 \mathrm{~mm}$ bore $4.7 \mathrm{~T}$ systems (gradient coil dimensions in both cases: OD $205 \mathrm{~mm}$, ID $120 \mathrm{~mm}$ ). Although it is not possible to generalize these results to all MRI scanners, given the vast array of different set-ups, our findings suggest that improved detection of iron oxide particles will be obtained by working at 7.0 $\mathrm{T}$ compared to lower field strengths. Beyond this field strength, the negative impact of field inhomogeneities and system constraints may counteract the benefits of higher field.

It has been shown that in response to disease, injury, and inflammation, VCAM-1 upregulates rapidly on activated 

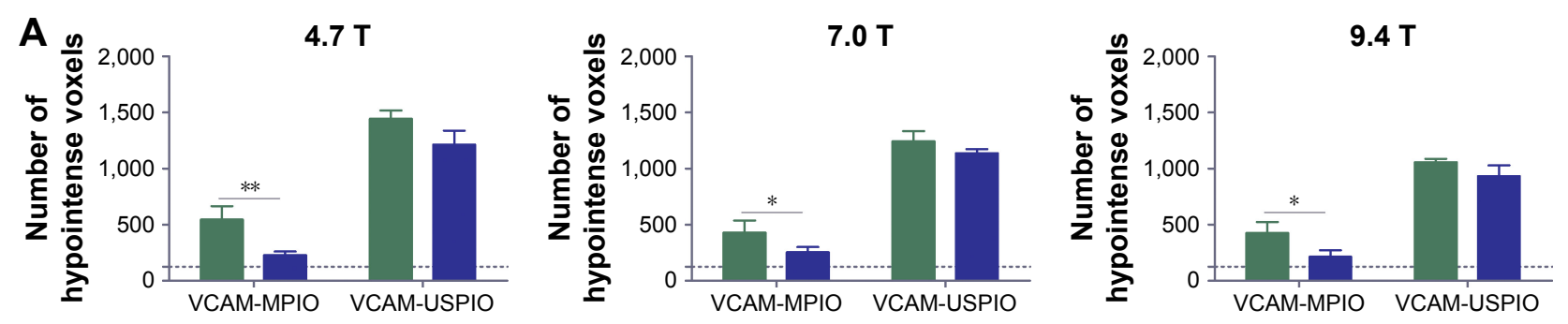

B

VCAM-MPIO

VCAM-USPIO
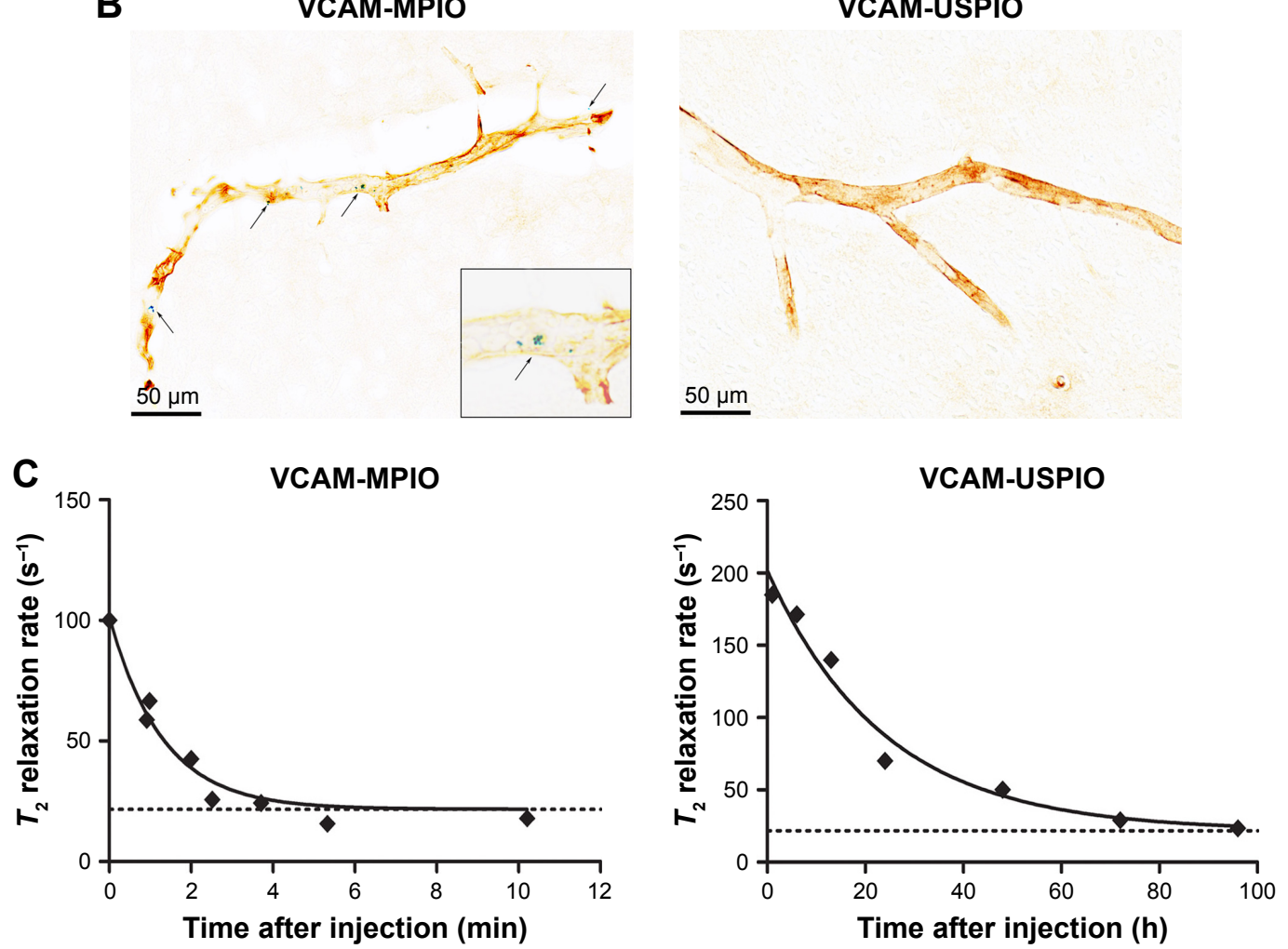

D

$1 \mathrm{~h}$

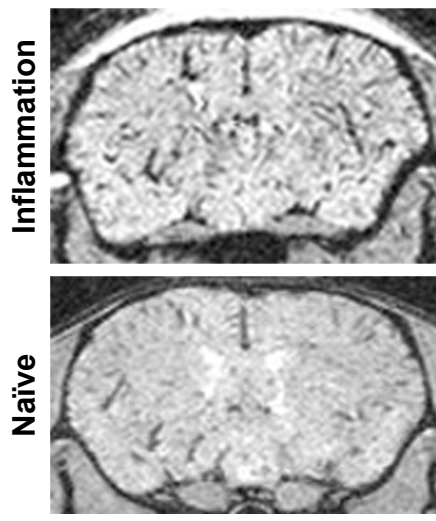

$13 \mathrm{~h}$
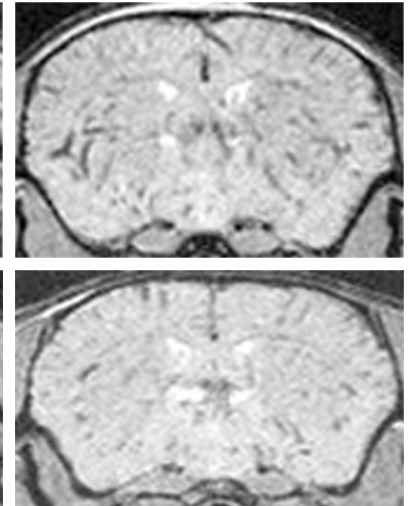

$80 \mathrm{~h}$
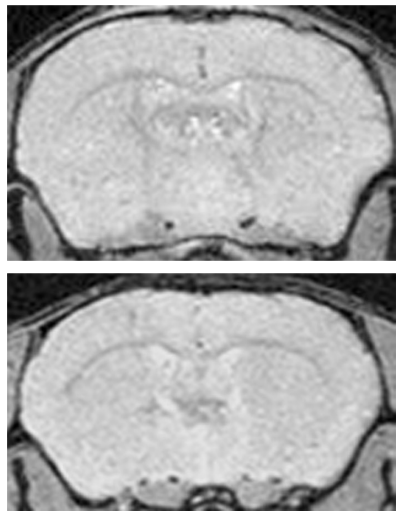

Figure 4 (A) Graph showing quantitation of MPIO- and USPIO-induced hypointensities in injected (green bar) and contralateral (blue bar) hemispheres using MGE3D sequence. Baseline level of endogenous hypointensities is shown with the dotted line. A significant difference was evident between the 2 hemispheres for VCAM-MPIOinjected mice at all field strengths. No significant difference was found for VCAM-USPIO. N=4 per group; $* P<0.05$, $* * P<0.0$ I. (B) Histological staining for VCAM-I (brown) and iron beads (Perls' Prussian blue) demonstrating upregulation of VCAM-I on endothelial cells together with VCAM-MPIO binding (arrows). VCAM-USPIO was not detected in brain samples after perfusion, error bar=50 um (C) Graph showing the decay curve of the biological clearance of VCAM-MPIO and VCAM-USPIO, at a constant dose of $4 \mathrm{mg} \mathrm{Fe} / \mathrm{kg}$ of body weight over time after injection. Graph shows $T_{2}$ relaxivity values of blood samples at different time points after administration. Curve is fitted as a single-phase exponential decay constrained to a plateau at the average naïve blood sample values (baseline; dotted lines). (D) MGE3D images of mice injected with VCAMUSPIO at different time-points acquired at 7.0 T - images show the presence of VCAM-USPIO in an inflammation model (top row) and naïve mouse (bottom row) at I and $13 \mathrm{~h}$ after contrast agent administration. No signal was detected $80 \mathrm{~h}$ after injection in both cohorts.

Abbreviations: MGE3D, multi-gradient echo 3D; MPIO, microparticles of iron oxide; USPIO, ultra-small superparamagnetic iron oxide; VCAM, vascular cell adhesion molecule. 
endothelial cells of the brain. ${ }^{69}$ VCAM-1-targeted MPIO have been widely studied by a number of groups for detection of VCAM-1 in preclinical models of disease and have shown high sensitivity and specificity for detecting this biomarker in vivo. ${ }^{25,33-35,37,70-73}$ As a simple model for inducing endothelial activation, we have previously demonstrated that a focal unilateral injection of IL-1 $\beta$ causes unilateral hemispheric upregulation of adhesion molecules on the endovascular surface, including VCAM-1. ${ }^{25,70} \mathrm{We}$, therefore, used this model to determine the relative sensitivity and specificity of VCAM-MPIO and VCAM-USPIO for detection of target expression in vivo and found dramatically different results. For VCAM-MPIO, a combination of high valency targeted delivery of a high payload of iron oxide to sites of inflammation, and rapid clearance of MPIO from the blood conferred a high degree of specificity and sensitivity for target detection, as reported previously. . $^{25,30,33,39}$ These properties are crucial in detecting biomarkers such as VCAM-1, that have relatively low abundance on endothelial cells ${ }^{74}$ and may only be detectable using particles with higher contrast owing to their greater iron payload.

In contrast to VCAM-MPIO, no target-specific binding was detected by either MRI or histology (using Perls' Prussian blue staining) in mice injected with VCAM-USPIO. Moreover, our data show that VCAM-USPIO were still present in the blood circulation $13 \mathrm{~h}$ post-administration, in both naïve and IL-1 $\beta$ injected mice. As a consequence of this long circulation time, marked background contrast effects were evident, which preclude detection of target-specific binding in the injected hemisphere. This is not to say that target-specific binding is entirely absent in the case of VCAM-USPIO, but rather that it is undetectable owing to the continuing presence of circulating agent and the low relaxivity, and hence CNR, of the particles that are bound. Although, theoretically, it may be possible to identify an imaging window at which point all circulating USPIO have been eliminated, it is unlikely that specific binding will still be present and detectable at that time point. Moreover, the necessity for a long time window between contrast administration and imaging would also be less favorable for downstream clinical use, than an agent that allowed administration and rapid imaging within a short timeframe thereafter. In practice, we were unable to detect any VCAM-USPIO binding on the activated endothelium $13 \mathrm{~h}$ after injection histologically, and no specific VCAM-USPIO contrast effects were visible by MRI at $80 \mathrm{~h}$ after injection when the blood pool has cleared.

These findings demonstrate empirically that MPIO provide significant benefits for molecularly targeted MRI in brain and exhibit higher sensitivity than USPIO. While for USPIO many effective applications have been reported, the small size of these particles is likely to be limiting for their application in molecular MRI studies, particularly of endovascular targets. One of the drawbacks of MPIO that is often discussed, is their limited translational capacity to the clinic. However, with the new generation of degradable biocompatible particles, ${ }^{30}$ this platform now has realizable translational potential.

\section{Conclusion}

The overall goal of this work was to identify the optimal pulse sequence, field strength, and particle size for molecular MRI in mice. Our results demonstrate clear advantages of the MGE3D sequence over bSSFP3D and GE3D for detection of iron oxide particles in vivo. Further, the specificity and sensitivity of VCAM-MPIO, compared with VCAM-USPIO, for delineation of activated endothelium in a mouse model of acute inflammation has been shown. With advances in the generation of biodegradable micrometer-sized iron oxide particles, these agents look promising for clinical translation in a broad range of central nervous system diseases, including inflammation and cancer.

\section{Acknowledgments}

This work was funded by Cancer Research UK (grant number C5255/A15935 to NRS). The authors would like to thank Karla Miller, Paul Kinchesh, Sean Smart, and Emeline Ribot for assistance in MRI sequence development. NZ was funded by a studentship from the CRUK \& EPSRC Cancer Imaging Centre, in Oxford, UK (grant number C5255/A16466), and a Radcliffe Scholarship from University College, Oxford, UK.

\section{Author contributions}

NZ and NRS proposed and designed the study. NZ performed the experiments and data analysis. AAK developed sequences and image post-processing algorithms. FP-B developed and guided the generation of conjugates. LB assisted with physiochemical properties of conjugates. JRL assisted with blood half-life measurements. MSS assisted with the animal model. NRS supervised assembly of manuscript and NZ wrote the manuscript. All authors contributed to drafting and editing the manuscript and agreed to be accountable for all aspects of the work. All authors contributed toward data analysis, drafting and revising the paper and agree to be accountable for all aspects of the work.

\section{Disclosure}

The authors report no conflicts of interest in this work. 


\section{References}

1. Bulte JW, Kraitchman DL. Iron oxide MR contrast agents for molecular and cellular imaging. NMR Biomed. 2004;17(7):484-499.

2. Korchinski DJ, Taha M, Yang R, Nathoo N, Dunn JF. Iron oxide as an MRI contrast agent for cell tracking. Magn Reson Insights. 2015; 8(Suppl 1):15-29.

3. Dodd SJ, Williams M, Suhan JP, Williams DS, Koretsky AP, Ho C. Detection of single mammalian cells by high-resolution magnetic resonance imaging. Biophys J. 1999;76(1 Pt 1):103-109.

4. Gupta AK, Gupta M. Synthesis and surface engineering of iron oxide nanoparticles for biomedical applications. Biomaterials. 2005;26(18): 3995-4021.

5. Stoll G, Bendszus M. New approaches to neuroimaging of central nervous system inflammation. Curr Opin Neurol. 2010;23(3):282-286.

6. Shapiro EM, Skrtic S, Sharer K, Hill JM, Dunbar CE, Koretsky AP. MRI detection of single particles for cellular imaging. Proc Natl Acad Sci U S A. 2004;101(30):10901-10906.

7. Weissleder R, Bogdanov A, Neuwelt EA, Papisov M. Long-circulating iron oxides for MR imaging. Adv Drug Deliv Rev. 1995;16(2-3): 321-334.

8. Deckers F, Corthouts B, Nackaerts Y, Ozsarlak O, Parizel PM, De Schepper AM. Original article the influence of MR field strength on the detection of focal liver lesions with superparamagnetic iron oxide. Eur Radiol. 1997;7(6):887-892.

9. Farrar CT, Dai G, Novikov M, et al. Impact of field strength and iron oxide nanoparticle concentration on the linearity and diagnostic accuracy of off-resonance imaging. NMR Biomed. 2009;21(5):453-463.

10. Tanimoto A, Kuribayashi S. Application of superparamagnetic iron oxide to imaging of hepatocellular carcinoma. Eur J Radiol. 2006; 58(2):200-216.

11. Girard OM, Du J, Agemy L, et al. Optimization of iron oxide nanoparticle detection using ultrashort echo time pulse sequences: comparison of T1, T2*, and synergistic T1-T2* contrast mechanisms. Magn Reson Med. 2011;65(6):1649-1660.

12. Kim SH, Oh SN, Choi HS, et al. USPIO enhanced lymph node MRI using 3D multi-echo GRE in a rabbit model. Contrast Media Mol Imaging. 2016;11(6):544-549.

13. Zheng L, Zhang Z, Khazaie K, et al. MRI-monitored intra-tumoral injection of iron-oxide labeled Clostridium novyi-NT anaerobes in pancreatic carcinoma mouse model. PLoS One. 2014;9(12):e116204.

14. Wei H, Bruns OT, Kaul MG, et al. Exceedingly small iron oxide nanoparticles as positive MRI contrast agents. Proc Natl Acad Sci US A. 2017; 114(9):2325-2330.

15. Modo MMJ, Bulte JWM. Molecular and Cellular MR Imaging. Boca Raton: CRC Press; 2007;4(3):458.

16. Christen T, Pannetier NA, Ni W, et al. MR vascular fingerprinting: a new approach to compute cerebral blood volume, mean vessel radius, and oxygenation maps in the human brain. Neuroimage. 2014;89:262-270.

17. Qui D, Zaharchul G, Christen T, Ni WW, Moseley ME. ContrastEnhanced Functional Blood Volume Imaging (CE-fBVI): enhanced sensitivity for brain activation in humans using the ultrasmall superparamagnetic iron oxide agent ferumoxytol. Neuroimage. 2012;62(3): 1726-1731.

18. Neuwelt EA, Várallyay P, Bagó AG, Muldoon LL, Nesbit G, Nixon R. Imaging of iron oxide nanoparticles by MR and light microscopy in patients with malignant brain tumours. Neuropathol Appl Neurobiol. 2004;30(5):456-471.

19. Neuwelt EA, Hamilton BE, Varallyay CG, et al. Ultrasmall superparamagnetic iron oxides (USPIOs): a future alternative magnetic resonance (MR) contrast agent for patients at risk for nephrogenic systemic fibrosis (NSF)? Kidney Int. 2009;75(5):465-474.

20. Weinstein JS, Varallyay CG, Dosa E, et al. Superparamagnetic iron oxide nanoparticles: diagnostic magnetic resonance imaging and potential therapeutic applications in neurooncology and central nervous system inflammatory pathologies, a review. J Cereb Blood Flow Metab. 2010; 30(1):15-35.
21. Wang YX. Superparamagnetic iron oxide based MRI contrast agents: current status of clinical application. Quant Imaging Med Surg. 2011; $1(1): 35-40$.

22. Motomura K, Ishitobi M, Komoike Y, et al. SPIO-enhanced magnetic resonance imaging for the detection of metastases in sentinel nodes localized by computed tomography lymphography in patients with breast cancer. Ann Surg Oncol. 2011;18(12):3422-3429.

23. Pouw JJ, Grootendorst MR, Bezooijen R, et al. Pre-operative sentinel lymph node localization in breast cancer with superparamagnetic iron oxide MRI: the SentiMAG multicentre trial imaging subprotocol. $\mathrm{BrJ}$ Radiol. 2015;88(1056):20150634.

24. Shapiro EM, Skrtic S, Koretsky AP. Sizing it up: cellular MRI using micron-sized iron oxide particles. Magn Reson Med. 2005;53(2): 329-338.

25. McAteer MA, Sibson NR, von Mühlen C. In vivo magnetic resonance imaging of acute brain inflammation using microparticles of iron oxide. Nat Med. 2007;13(10):1253-1258.

26. Chaumeil MM, Gini B, Yang H, et al. Longitudinal evaluation of MPIO-labeled stem cell biodistribution in glioblastoma using high resolution and contrast-enhanced MR imaging at 14.1 tesla. Neuro Oncol. 2012;14(8):1050-1061.

27. Serres S, Mardiguian S, Campbell SJ. VCAM-1-targeted magnetic resonance imaging reveals subclinical disease in a mouse model of multiple sclerosis. FASEB J. 2011;25(12):4415-4422.

28. Murrell DH, Zarghami N, Jensen MD, et al. MRI surveillance of cancer cell fate in a brain metastasis model after early radiotherapy. Magn Reson Med. 2017;78(4):1506-1512.

29. Murrell DH, Zarghami N, Jensen MD, Chambers AF, Wong E, Foster PJ. Evaluating changes to blood-brain barrier integrity in brain metastasis over time and after radiation treatment. Transl Oncol. 2016;9(3): 219-227.

30. Perez-Balderas F, van Kasteren SI, Aljabali AA, et al. Covalent assembly of nanoparticles as a peptidase-degradable platform for molecular MRI. Nat Commun. 2017;8:14254

31. Ahmed N, Ahmad NM, Fessi H, Elaissari A. In vitro MRI of biodegradable hybrid (iron oxide/polycaprolactone) magnetic nanoparticles prepared via modified double emulsion evaporation mechanism. Colloids Surf B Biointerfaces. 2015;130:264-271.

32. Ahmed N, Jaafar-Maalej C, Eissa MM, Fessi H, Elaissari A. New oil-in-water magnetic emulsion as contrast agent for in vivo magnetic resonance imaging (MRI). J Biomed Nanotechnol. 2013;9(9): 1579-1585.

33. Serres S, Soto MS, Hamilton A, et al. Molecular MRI enables early and sensitive detection of brain metastases. Proc Natl Acad Sci US A. 2012; 109(17):6674-6679.

34. Hoyte LC, Brooks KJ, Nagel S, et al. Molecular magnetic resonance imaging of acute vascular cell adhesion molecule-1 expression in a mouse model of cerebral ischemia. J Cereb Blood Flow Metab. 2010; 30(6):1178-1187.

35. Evans MC, Serres S, Khrapitchev AA, et al. $\mathrm{T}_{2}$-weighted MRI detects presymptomatic pathology in the SOD1 mouse model of ALS. J Cereb Blood Flow Metab. 2014;34(5):785-793.

36. Gauberti M, Montagne A, Marcos-Contreras OA, Le Béhot A, Maubert E, Vivien D. Ultra-sensitive molecular MRI of vascular cell adhesion molecule-1 reveals a dynamic inflammatory penumbra after strokes. Stroke. 2013;44(7):1988-1996.

37. Montagne A, Gauberti M, Macrez R, et al. Ultra-sensitive molecular MRI of cerebrovascular cell activation enables early detection of chronic central nervous system disorders. Neuroimage. 2012;63(2): 760-770.

38. Yang Y, Yang Y, Yanasak N, Schumacher A, Hu TC. Temporal and noninvasive monitoring of inflammatory-cell infiltration to myocardial infarction sites using micrometer-sized iron oxide particles. Magn Reson Med. 2010;63(1):33-40.

39. Gauberti M, Montagne A, Quenault A, Vivien D. Molecular magnetic resonance imaging of brain-immune interactions. Front Cell Neurosci. 2014;8:389. 
40. McAteer MA, Mankia K, Ruparelia N, et al. A leukocyte-mimetic magnetic resonance imaging contrast agent homes rapidly to activated endothelium and tracks with atherosclerotic lesion macrophage content. Arterioscler Thromb Vasc Biol. 2012;32(6):1427-1435.

41. Heyn C, Bowen CV, Rutt BK, Foster PJ. Detection threshold of single SPIO-labeled cells with FIESTA. Magn Reson Med. 2005;53(2): 312-320.

42. Shapiro EM, Koretsky AP. Micron-sized iron oxide particles (MPIOs) for cellular imaging: more bang for the buck. Nanoparticles Biomed Imaging. Fundamental Biomedical Technologies, Springer. 2008; 102:141-161.

43. Tassa C, Duffner JL, Lewis TA, et al. Binding affinity and kinetic analysis of targeted small molecule-modified. 2010;21(1):14-19.

44. Liu L, Hitchens TK, Ye Q, et al. Decreased reticuloendothelial system clearance and increased blood half-life and immune cell labeling for nano- and micron-sized superparamagnetic iron-oxide particles upon pre-treatment with Intralipid. Biochim Biophys Acta. 2013; 1830(6):3447-3453.

45. Mabray MC, Lillaney P, Sze CH, et al. In vitro capture of small ferrous particles with a magnetic filtration device designed for intravascular use with intraarterial chemotherapy: proof-of-concept Study. J Vasc Interv Radiol. 2016;27(3):426-432.

46. Bayburtskiy F, Semenova G, Brusentsov N, et al. Studying of a Magnetic Resonance in Contrasting Agents on the Basis of Biodecomposed Magnetic Fluid. Oxidation Communications. 2007;30(2):466-471.

47. Shen T, Weissleder R, Papisov M, Bogdanov A Jr, Brady TJ. Monocrystalline iron oxide nanocompounds (MION): physicochemical properties. Magn Reson Med. 1993;29(5):599-604.

48. Pittet MJ, Swirski FK, Reynolds F, Josephson L, Weissleder R. Labeling of immune cells for in vivo imaging using magnetofluorescent nanoparticles. Nat Protoc. 2006;1(1):73-79.

49. Bisschops MAT, Luyben KCAM, Van Der Wielen LAM. Generalized Maxwell-Stefan approach for swelling kinetics of dextran gels. Ind Eng Chem Res. 1998;37(8):3312-3322.

50. Wunderbaldinger P, Josephson L, Weissleder R. Tat peptide directs enhanced clearance and hepatic permeability of magnetic nanoparticles. Bioconjug Chem. 2002;13(2):264-268.

51. Kelly KA, Allport JR, Tsourkas A, Shinde-Patil VR, Josephson L, Weissleder R. Detection of vascular adhesion molecule-1 expression using a novel multimodal nanoparticle. Circ Res. 2005;96(3): 327-336.

52. Kim SH, Choi D, Lim JH, et al. Optimal pulse sequence for ferumoxides-enhanced MR imaging used in the detection of hepatocellular carcinoma: a comparative study using seven pulse sequences. Korean J Radiol. 2002;3(2):87-97.

53. Schieda N, Avruch L, Shabana WM, Malone SC. Multi-echo gradient recalled echo imaging of the pelvis for improved depiction of brachytherapy seeds and fiducial markers facilitating radiotherapy planning and treatment of prostatic carcinoma. JMagn Reson Imaging. 2015;41(3):715-720.

54. Martin N, Malfair D, Zhao Y, et al. Comparison of MERGE and axial T2-weighted fast spin-echo sequences for detection of multiple sclerosis lesions in the cervical spinal cord. AJR Am J Roentgenol. 2012; 199(1):157-162

55. Melemenidis S, Jefferson A, Ruparelia N, et al. Molecular magnetic resonance imaging of angiogenesis in vivo using polyvalent cyclic RGD-iron oxide microparticle conjugates. Theranostics. 2015;5(5): 515-529.

56. Bangerter NK, Hargreaves BA, Vasanawala SS, Pauly JM, Gold GE, Nishimura DG. Analysis of multiple-acquisition SSFP. Magn Reson Med. 2004;51(5):1038-1047.
57. Ribot EJ, Wecker D, Trotier AJ, et al. Water selective imaging and bSSFP banding artifact correction in humans and small animals at 3T and 7T, respectively. PLoS One. 2015;10(10):e0139249.

58. Chen S, Reynolds F, Yu L, Weissleder R, Josephson L. A screening paradigm for the design of improved polymer-coated superparamagnetic iron oxide nanoparticles. J Mater Chem. 2009;19(35):6387.

59. Delangre S, Vuong QL, Henrard D, Po C, Gallez B, Gossuin Y. Bottomup study of the MRI positive contrast created by the Off-Resonance saturation sequence. J Magn Reson. 2015;254:98-109.

60. Reimer P, Balzer T. Ferucarbotran (Resovist): a new clinically approved RES-specific contrast agent for contrast-enhanced MRI of the liver: properties, clinical development, and applications. Eur Radiol. 2003;13(6):1266-1276.

61. Klohs J, Deistung A, Ielacqua GD, et al. Quantitative assessment of microvasculopathy in arcA $\beta$ mice with USPIO-enhanced gradient echo MRI. J Cereb Blood Flow Metab. 2016;36(9):1614-1624.

62. van der Kouwe AJW, Benner T, Salat DH, Fischl B. Brain morphometry with multiecho MPRAGE. Neuroimage. 2008;40(2):559-569.

63. Choi JS, Kim MJ, Kim JH, et al. Comparison of multi-echo and singleecho gradient-recalled echo sequences for SPIO-enhanced liver MRI at 3 T. Clin Radiol. 2010;65(11):916-923.

64. Mallett CL, Foster PJ. Optimization of the balanced steady state free precession (bSSFP) pulse sequence for magnetic resonance imaging of the mouse prostate at 3T. PLoS One. 2011;6(4):e18361.

65. Scheffler K, Lehnhardt S. Principles and applications of balanced SSFP techniques. Eur Radiol. 2003;13(11):2409-2418.

66. Huang SY, Seethamraju RT, Patel P, Hahn PF, Kirsch JE, Guimaraes AR. Body MR Imaging: Artifacts, k-Space, and Solutions. RadioGraphics. 2015;35(5):1439-1460.

67. DiFrancesco MW, Rasmussen JM, Yuan W, et al. Comparison of SNR and CNR for in vivo mouse brain imaging at 3 and $7 \mathrm{~T}$ using well matched scanner configurations. Med Phys. 2008;35(9):3972-3978.

68. Biswas J, Nelson CB, Runge VM, et al. Brain tumor enhancement in magnetic resonance imaging: comparison of signal-to-noise ratio (SNR) and contrast-to-noise ratio (CNR) at 1.5 versus 3 tesla. Invest Radiol. 2005;40(12):792-797.

69. Ley K, Laudanna C, Cybulsky MI, Nourshargh S. Getting to the site of inflammation: the leukocyte adhesion cascade updated. Nat Rev Immunol. 2007;7(9):678-689.

70. Sibson NR, Blamire AM, Bernades-Silva M, et al. MRI detection of early endothelial activation in brain inflammation. Magn Reson Med. 2004;51(2):248-252.

71. Serres S, Mardiguian S, Campbell SJ, et al. VCAM-1-targeted magnetic resonance imaging reveals subclinical disease in a mouse model of multiple sclerosis. FASEB J. 2011;25(12):4415-4422.

72. Mardiguian S, Serres S, Ladds E, et al. Anti-IL-17A treatment reduces clinical score and VCAM-1 expression detected by in vivo magnetic resonance imaging in chronic relapsing EAE ABH mice. Am J Pathol. 2013;182(6):2071-2081.

73. Akhtar AM, Schneider JE, Chapman SJ, et al. In vivo quantification of VCAM-1 expression in renal ischemia reperfusion injury using non-invasive magnetic resonance molecular imaging. PLoS One. 2010;5(9):e12800.

74. Jefferson A, Wijesurendra RS, McAteer MA, et al. Molecular imaging with optical coherence tomography using ligand-conjugated microparticles that detect activated endothelial cells: rational design through target quantification. Atherosclerosis. 2011;219(2):579-587. 


\section{Supplementary materials}

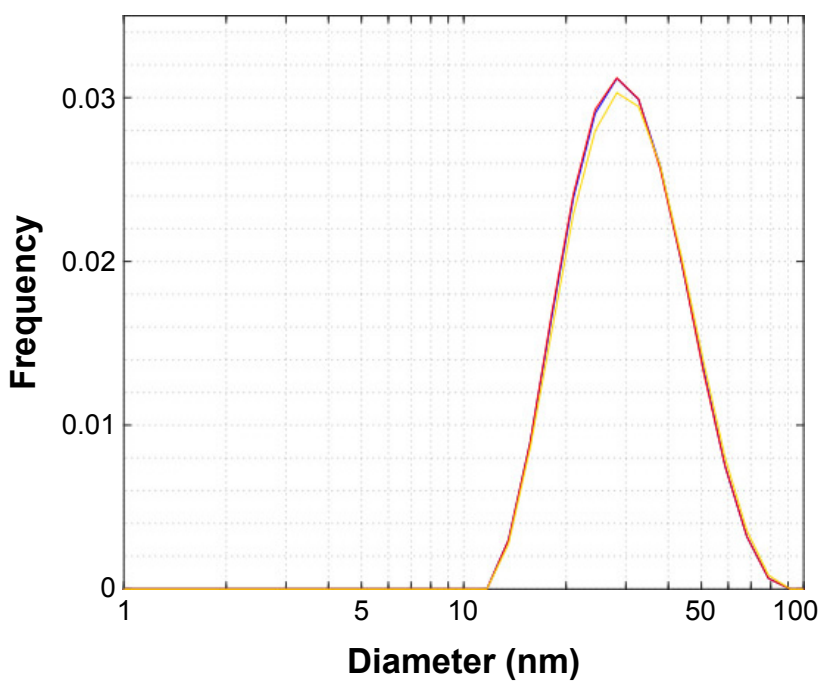

Figure SI Particle size distribution (intensity-weighted) of VCAM-USPIO measured by DLS.

Note: The 3 traces are repeated measurements of the same sample.

Abbreviations: DLS, dynamic light scattering; USPIO, ultra-small superparamagnetic iron oxide; VCAM, vascular cell adhesion molecule.

A
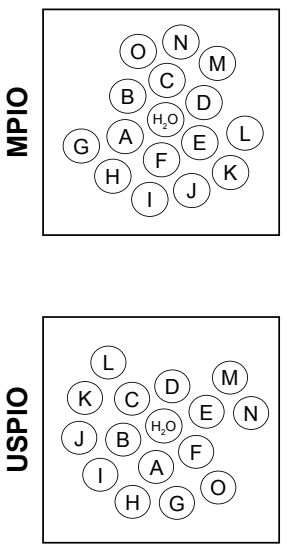

$T_{2}(\mathrm{~ms})$

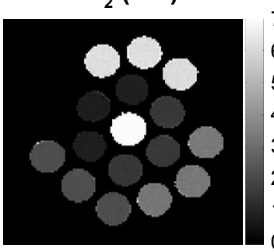

$T_{2}(\mathrm{~ms})$

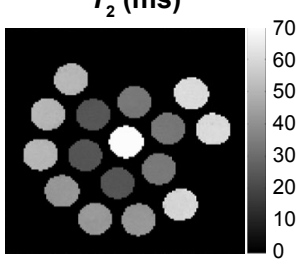

$R_{2}(\mathrm{~Hz})$

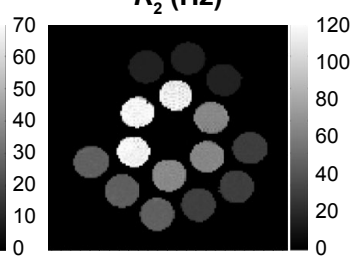

$R_{2}(\mathrm{~Hz})$

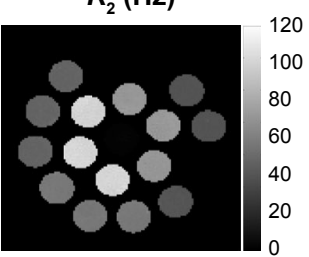

B

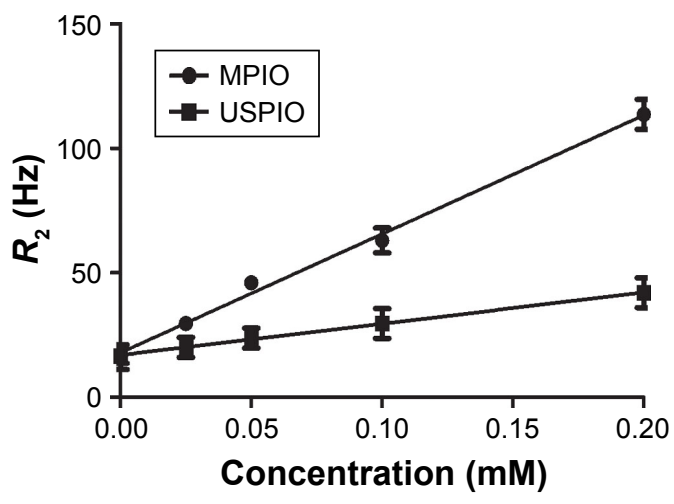

Figure S2 (A) MPIO (top row) and USPIO (bottom row) were embedded in $2 \%$ agarose gel at the same iron concentration (triplicates). (A-C): $0.2 \mathrm{mM}$, (D-F): $0.1 \mathrm{mM}$, (G-I): $0.05 \mathrm{mM},(\mathrm{J}-\mathrm{L}): 0.024 \mathrm{mM}$, and (M-O): PBS. $T_{2}(\mathrm{~ms})$ and $R_{2}(\mathrm{~Hz})$ maps generated at $7.0 \mathrm{~T}$ are shown. (B) $T_{2}$ relaxivity was measured for both particles; MPIO showed significantly greater relaxivity (steeper slope; $P<0.000 \mathrm{I}$ ) than USPIO. Errors are expressed as mean \pm SD for $\mathrm{n}=3$.

Abbreviations: MPIO, microparticles of iron oxide; PBS, phosphate buffered saline; USPIO, ultra-small superparamagnetic iron oxide. 

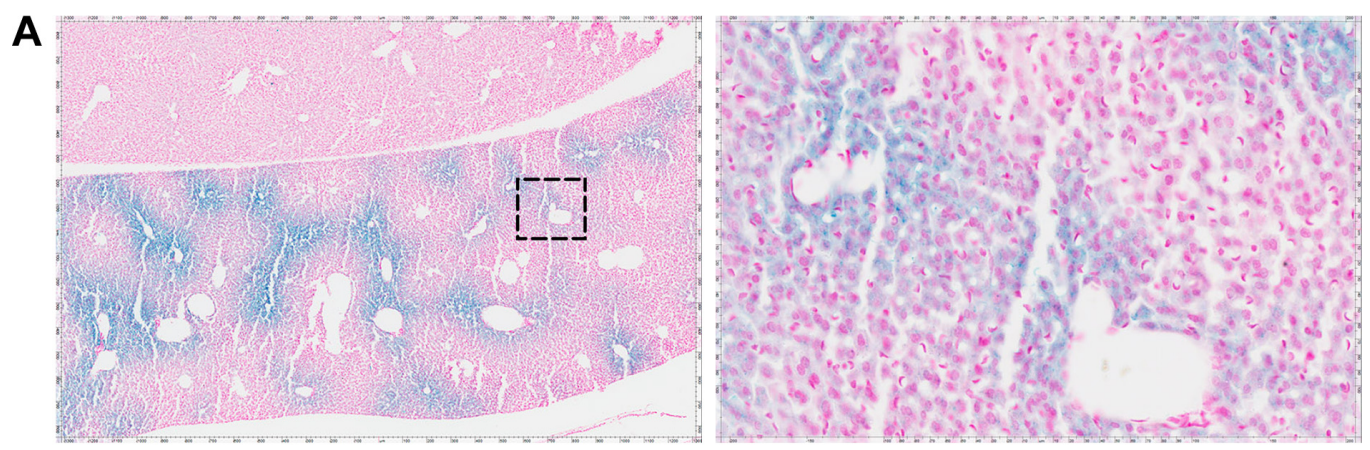

B
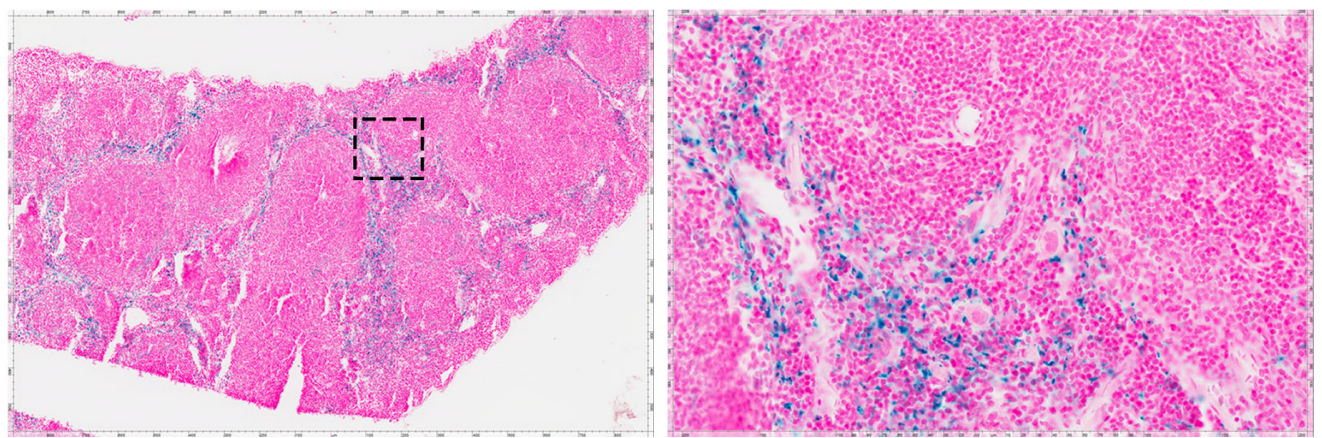

Figure S3 Positive control Prussian blue staining for USPIO and counterstaining with nuclear fast red.

Notes: (A) Mouse liver section, blue: USPIO; pink: nuclei, (B) Mouse spleen section, blue: USPIO; pink: nuclei.

Abbreviation: USPIO, ultra-small superparamagnetic iron oxide.

\section{Publish your work in this journal}

The International Journal of Nanomedicine is an international, peerreviewed journal focusing on the application of nanotechnology in diagnostics, therapeutics, and drug delivery systems throughou the biomedical field. This journal is indexed on PubMed Central, MedLine, CAS, SciSearch $®$, Current Contents $\AA /$ Clinical Medicine,
Journal Citation Reports/Science Edition, EMBase, Scopus and the Elsevier Bibliographic databases. The manuscript management system is completely online and includes a very quick and fair peer-review system, which is all easy to use. Visit http://www.dovepress.com/ testimonials.php to read real quotes from published authors. 